\title{
CaseFinder: A Flexible Real-time Online Surveillance Registry for Infectious Disease Physicians to Report Cases of Carbapenem-resistant Enterobacteriaceae (CRE)
}

\author{
Donald Curtis*1, Scott Weissman², Dimitri Drekonja ${ }^{3}$, Susan Beekmann ${ }^{4}$, Benjamin \\ Buckmaster $^{1}$, John Lynch ${ }^{5}$, April Abbott ${ }^{6}$, Ferric Fang ${ }^{6}$ and Philip Polgreen ${ }^{4}$
}

${ }^{1}$ Coe College, Cedar Rapids, IA, USA; ${ }^{2}$ Seattle Children's Hospital, Seattle, WA, USA; ${ }^{3}$ Minneapolis Veterans Affairs Medical Center, Minneapolis, MN, USA; ${ }^{4}$ University of lowa, lowa City, IA, USA; ${ }^{5}$ Harborview Medical Center, Seattle, WA, USA; ${ }^{6}$ University of Washington, Seattle, WA, USA

\section{Objective}

To create a flexible online surveillance system for infectious disease experts to report cases of emerging infectious diseases.

\section{Introduction}

The Infectious Disease Society of America's Emerging Infections Network (EIN) is a sentinel network of over 1,200 practicing infectious disease physicians, supported by the Centers for Disease Control and Prevention (CDC). In January 2012, the EIN listserv fielded a member inquiry about treatment recommendations for a complicated polymicrobial wound infection in a traveler returning to the United States from India. The posting led to a member-to-member communication that resulted in shipment of clinical microbiology isolates from one member's hospital to another's research laboratory. Molecular evaluation of the clinical isolates uncovered previously undetected carriage of the emerging NDM-1 enzyme in 2 of the Enterobacteriaceae species. Based on this interaction, we built a flexible online surveillance registry (CaseFinder) for infectious disease physicians to report cases of CRE.

\section{Methods}

To ascertain the frequency and nature of CRE infections treated by EIN members, a survey was sent to EIN members in July 2012 that elicited risk factors and clinical features associated with CRE. Survey opt-out items also allowed respondents to specify that they had not treated any CRE infections. Concurrently, we developed a formal relational data model for CRE infection survey data, allowing for analysis and visualization. The data model was implemented in Python using the Object-Relational Mapping provided by the Django web framework, which we used to implement the backend server component to the online registry. An interactive front-end web application, written in Javascript using the jQuery library, retrieves data via the AJAX web protocol. Geolocated data is visualized using the OpenLayers library to render map tiles and provide interactive controls such as panning and zooming.

\section{Results}

The crowd-sourced online registry for infectious disease experts to report CRE infections, called CaseFinder (http://casefinder.org/), was developed, released, and seeded with data from the EIN survey. To date, a total of 69 cases have been submitted, describing 53 infections with Klebsiella pneumoniae, 7 with Escherichia coli and 9 with other Enterobacteriaceae, representing 7 of 9 US Census divisions. Another 214 members have indicated that they have not seen any cases to date. CaseFinder includes: an online data entry component (to supplant the original EIN listserv survey); real-time filtering of data; and interactive maps that geolocate survey responses using the first 2 digits of the treating facility's zip code. Users can filter data based on species, clinical features (age, gender), resistance profile, or 2-digit zip code. CaseFinder can also display clinical case data in an exportable line-item format.

\section{Conclusions}

We have created a web-based data registry for CRE infections in the US. Populated by EIN survey responses, the registry already has a collection 283 data points - 69 cases of CRE and 214 reports indicating the absence of cases - and is open for ongoing submission of data represented in real time. This system can serve as a de facto national surveillance system for CRE infections - an important but not yet universally reportable condition. Our platform can be expanded to map and track other emerging infections seen by infectious diseases physicians. We are currently working to incorporate molecular fingerprinting and typing information into the data model. The site will also provide incentives for infectious disease experts to submit cases in underrepresented geographic areas. In future efforts we will incorporate "machine learning" techniques to leverage knowledge from infectious disease experts on existing cases and provide features such as an intelligent automated alert system.

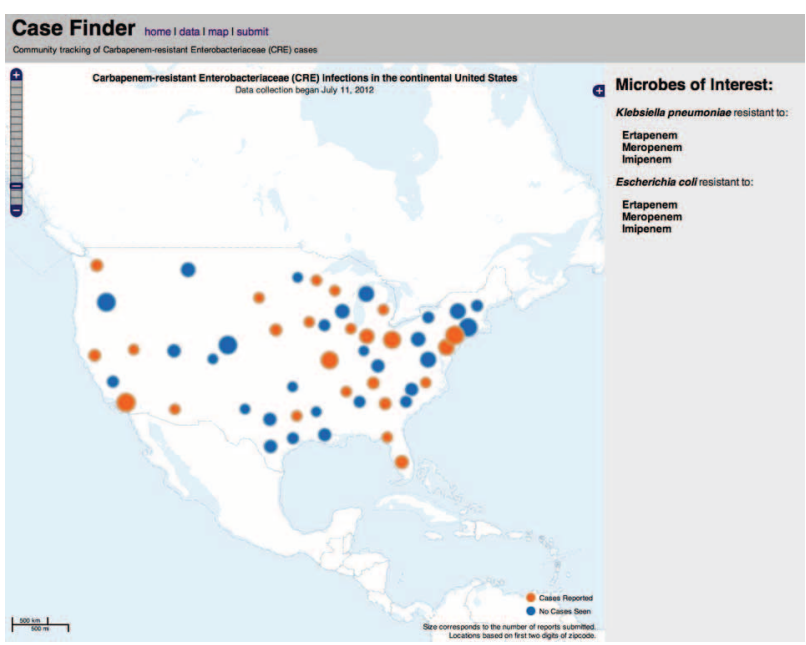

Keywords

Surveillance; Carbapenemases; Antibiotic resistance; Enterobacteriaceae; Klebsiella pneumoniae

\section{*Donald Curtis}

E-mail: dcurtis@coe.edu 